\title{
Palbociclib and letrozole for ER-positive, HER2-negative advanced breast cancer
}

$\mathrm{O}$ n February 3, 2015, the US Food and Drug Administration (FDA) approved palbociclib in combination with letrozole for first-line treatment of postmenopausal women with estrogen receptor (ER)-positive, human epidermal growth factor receptor 2 (HER2)-negative advanced breast cancer. The combination was reviewed and approved 2 months ahead of schedule under the FDA's breakthrough therapy designation and priority review program. Palbociclib is an oral small molecule inhibitor of cyclin-dependent kinases (CDK) 4 and 6, while letrozole is an aromatase inhibitor (AI), a type of endocrine therapy that reduces circulating estrogen levels (see sidebar). The 2 agents were shown to have potentially synergistic tumor growth inhibitory activity in preclinical models of ER-positive breast cancer.

Approval was based on the finding of improved progressionfree survival (PFS) in the international, multicenter, open-label, randomized phase 2 PALOMA-1/TRIO-18 study. Between December 2009 and May 2012, 165 postmenopausal women aged 18 years or older with ER-positive, HER2-negative locally recurrent or unresectable breast cancer, were recruited from 50 sites in 12 countries. Patients were enrolled in 2 separate cohorts, sequentially, on the basis of ER-positive, HER2-negative status alone (cohort $1 ; \mathrm{n}=66$ ) or in combination with cyclin $\mathrm{D}$ gene amplification or loss of p16 (cohort 2; n = 99). In both cohorts, patients were randomized 1:1 to $2.5 \mathrm{mg}$ letrozole once daily alone (81 patients) or in combination with $125 \mathrm{mg}$ palbociclib (84 patients) once daily for 3 weeks followed by 1 week off in 28-day cycles until disease progression, unacceptable toxicity, study withdrawal, or death.

Randomization was stratified by disease site (visceral, bone only, or other) and disease-free interval ( $>12$ months from end of adjuvant treatment to recurrence compared with $<12$ months or de novo metastatic disease). Eligible patients were required to have measurable disease by Response Evaluation Criteria in Solid Tumors (RECIST) or bone-only disease with Eastern Cooperative Oncology Group (ECOG) performance status of 0 or 1 and adequate organ function. Meanwhile, patients who were treated with neoadjuvant letrozole in the year before study enrollment, who had previously received CDK inhibitors, who had received any treatment for advanced breast cancer, or those with brain metastases were excluded.

A CT or MRI scan of the chest, abdomen, and pelvis; radiography of bone lesions; and clinical assessment of cutane-

\section{What's new, what's important}

Palbociclib is a novel CDK 4 and 6 inhibitor that recently received approval from the FDA in combination with letrzole for the treatment of postmenopausal women with estrogen receptor (ER)-positive, HER2-negative metastatic breast cancer. The agency approved a starting dose of palbociclib $125 \mathrm{mg}$ once daily taken with food for 21 days, followed by 7 days off treatment, repeating every 28 days along with letrozole $2.5 \mathrm{mg}$ daily.

In the randomized phase 2 study that formed the basis of the approval, grade $3(57 \%)$ or $4(5 \%)$ decreased neutrophil counts were reported in $57 \%$ and $5 \%$, respectively, of patients who received the palbociclib plus letrozole combination. Febrile neutropenia was also reported. So it is important to monitor blood counts before therapy initiation and at the beginning of each cycle of treatment with palbociclib and Day 14 of the first 2 cycles. For patients with grade 3 neutropenia, the blood count should be repeated 1 week later. Dose interruption, dose reduction, or delay in starting treatment cycles is recommended for patients who develop grade 3 or 4 neutropenia. Patients should be monitored for signs and symptoms of infection and treated as medically appropriate.

Higher incidence of pulmonary embolism (PE) has been reported in $5 \%$ patients who were treated with palbociclib plus letrozole, compared with no cases among patients who were treated with letrozole alone. As such, patients should also be monitored for signs and symptoms of PE and treatedas medically appropriate.

Many ongoing and completed studies will help us to understand the role of palbociclib in combination with other endocrine therapies in metastatic setting (PALOMA 3) and early breast cancer (NSABP B 54) and NSABP FB 11.

- Jame Abraham, MD, FACP (abrahaj5@ccf.org)

ous disease were performed at screening and every 8 weeks. Bone scans were performed at baseline and every 12 weeks, and hematological and blood chemistry analyses were done every 2 weeks for the first 2 cycles and at the beginning of each cycle from then on.

The study population was generally balanced for baseline demographics and established prognostic factors, including age (median, 63 vs 64 years for cohorts 1 and 2, respectively) and ECOG performance status ( 0 in $55 \%$ and $56 \%$ and 1 in $45 \%$ and 44\%, for cohorts 1 and 2, respectively),

Report prepared by Jane De Lartigue, PhD. JCSO 2015;13:83-86. @2015 Frontline Medical Communications. DOI 10.12788/icso.0113. 


\section{Mechanism of action: palbociclib and letrozole Inextricably linked CDK and ER pathways provide biological rationale}

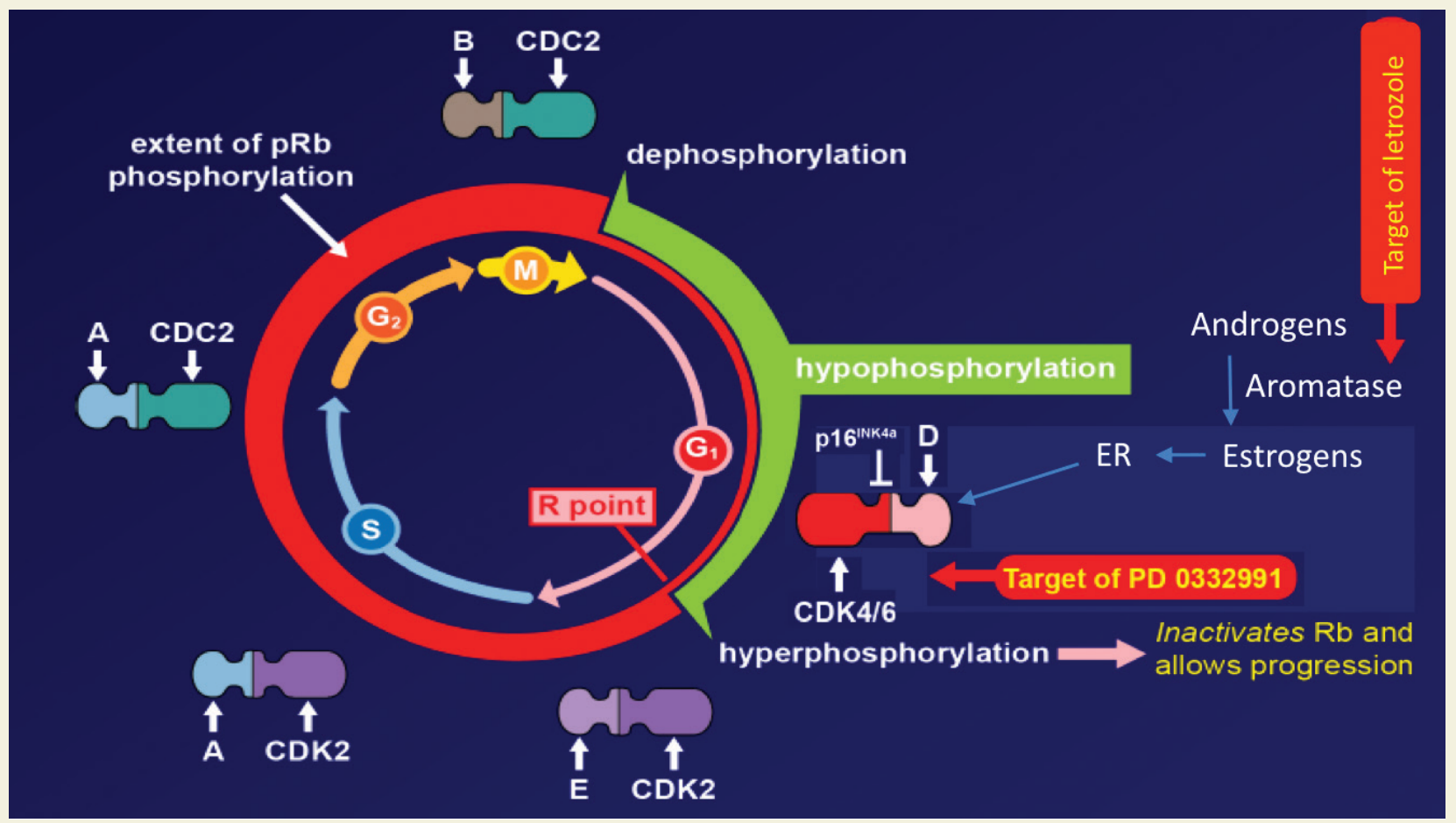

Figure reproduced and modified with permission from Dr RS Finn. ${ }^{1}$

Actively dividing eukaryotic cells pass through a highly regulated series of steps, known as the cell cycle, that culminate in their division into 2 identical daughter cells. Numerous cell cycle-associated proteins regulate the transition between phases of the cell cycle, ensuring each step occurs at the appropriate time. Among them are the cyclin-dependent kinases (CDKs), a family of serine/ threonine kinases that are themselves tightly regulated by associated activating or inhibitory proteins.

Palbociclib targets CDK4 and CDK6, the activity of which is regulated by cyclin DI and the CDK inhibitor p16. These particular CDKs have an important role in overseeing the transition between the first growth phase (GI) and the synthesis (S) phase of the cell cycle, a particularly important transition known as the restriction point, beyond which the cell commits to proceeding through the cell cycle regardless of external stimuli. CDK4/6 perform this function through the activation of downstream targets, primarily the retinoblastoma protein (pRb), which becomes hyperphosphorylated, releasing its suppression of transcription factors that control the G1/S transition.

Dysregulation of proteins like CDK4/6 and cyclin D1 is common in cancer and sustained activation of these proteins allows the cancer cell to continuously trigger the GI/S transition to enter the cell cycle, driving uncontrolled proliferation. The CDKs are readily druggable kinases and make attractive targets for cancer therapy; the clinical development of CDK inhibitors is most advanced in breast cancer. In preclinical studies, palbociclib demonstrated particularly potent effects in breast cancer cell lines that were estrogen receptor (ER)-positive. Endocrine therapies that reduce circulating levels of estrogen to block the cancer growth-promoting effects of this hormone in ER-positive tumors are standard of care for patients with this breast cancer subtype, but patients often develop resistance. Preclinical studies of palbociclib further demonstrated synergistic activity with endocrine therapy and in a model of acquired resistance to anti-estrogen therapy. As a downstream target of ER activation, dysregulation of the CDK pathway may represent a mechanism of resistance to endocrine therapy.

The PALOMA-1 study of palbociclib and letrozole provides proof-of-concept that the addition of a CDK4/6 inhibitor to an aromatase inhibitor, which blocks the activity of the enzyme aromatase that is responsible for converting androgens into estrogens, improves patient outcomes over letrozole alone. Mechanistic analyses demonstrated that the combination enhances the phosphorylation of $\mathrm{pRb}$, leading to a significant inhibition of downstream transcription factors such as E2F1 and FoxM, subsequent $\mathrm{G} 1$ cell cycle arrest, and reduced cell proliferation.

- Jane de Lartigue, PhD 


\section{News angle}

The accelerated approval by the US food and Drug Administration FDA of palbociclib with letrozole for advanced postmenopausal breast cancer was based on a randomized phase 2 study of 165 postmenopausal women with ER-positive, HER2-negative metastatic breast cancer, who had not been treated for advanced disease. Investigators found a progression-free survival rate of about 20.2 months among the women treated with the combination, compared with about 10.2 months among those treated with the aromatase inhibitor, letrozole.

The FDA noted in a February 3 statement announcing the approval that overall survival results are not yet available.

"The addition of palbociclib to letrozole provides a novel treatment option to women diagnosed with metastatic breast cancer," Dr Richard Pazdur, director of the Office of Hematology and Oncology Products in the FDA's Center for Drug Evaluation and Research, noted in the statement. He added that palbociclib had been approved under the FDA Accelerated Approval Program, which "allows approval of a drug to treat a serious or life-threatening disease based on clinical data showing the drug has an effect on a surrogate endpoint reasonably likely to predict clinical benefit to patients." Such an approval makes the drug available to patients earlier while the manufacturer conducts trials to confirm the benefits, according to the agency. The indications and usage sec- tion of the prescribing information includes the statement that "continued approval for this indication may be contingent upon verification and description of clinical benefit in a confirmatory trial."

In a statement released by the University of California, Los Angeles, Jonsson Comprehensive Cancer Center, the principal investigator of the study that led to the approval, Dr Richard S Finn, pointed out that palbociclib is the first drug in its class to be approved. In the same statement, Dr Dennis J. Slamon, director of the Revlon/UCLA Women's Cancer Research Program and Clini$\mathrm{cal} /$ Translational Research at the Jonsson Cancer Center, said he believes that palbociclib will become "a standard treatment approach for postmenopausal women with ER-positive and HER2negative metastatic breast cancer." He added that the magnitude of the benefit seen in the phase 2 study "was very gratifying and reminiscent of results we saw when we conducted the initial studies on Herceptin in HER2-positive breast cancers 2 decades ago."

Dr Finn reported the results of the phase II study at the annual meeting of the American Association for Cancer Research in April. $\mathrm{He}$ and Dr Slamon are investigators in the phase 3 confirmatory trial of palbociclib in over 600 women, which has completed enrollment, according to the UCLA release.

- Elizabeth Mechcatie but there were slight imbalances in disease site, disease-free interval, and previous treatment.

The primary endpoint of the study was PFS and, as of final analysis in November 2013, over a median follow-up of 29.6 months for the combination arm and 27.9 months for the letrozole alone group, median PFS was 20.2 months compared with 10.2 months, respectively (hazard ratio $[\mathrm{HR}], 0.488 ; P=.0004)$. Initially, analysis of PFS was intended to be based on cohort 2 alone, however, an unplanned interim analysis of cohort 1 suggested that it was unlikely that additional patient selection based on cyclin D1 amplification or p16 loss would improve outcomes over the use of ER-positive, HER2-negative status alone. As a result, accrual into cohort 2 was halted and PFS analysis was based on combined data from both cohorts. The authors conclude that ER-positivity remains the best and most effective biomarker for patient selection in this setting. In cohort 1, median PFS was 26.1 months in the combination arm, compared with 5.7 months in the letrozole arm (HR, 0.299; $P<.0001)$, whereas in cohort 2 it was 18.1 months compared with 11.2 months (HR, 0.508; $P=.0046)$.

A number of secondary endpoints also demonstrated significant improvement in the combination arm, including objective response (in both the intent to treat [ITT] population and among those with measurable disease), with a median duration of response of 20.3 months, and clinical benefit rate (in the ITT population). The study was not powered to detect overall survival (OS); few OS events had occurred at the time of final analysis, however, no detrimental effect on OS was observed.

Although more patients experienced adverse events (AEs) in the combination arm compared with those who received letrozole monotherapy (all patients in the combination arm had at least $1 \mathrm{AE}$ compared with $84 \%$ of patients treated with letrozole alone), the toxicity profile of the combination was found to be manageable and predictable. The most common AEs were neutropenia (28\% and $6 \%$ of grade 3 and 4, respectively), leucopenia (19\% grade 3 ), and fatigue ( $2 \%$ and $2 \%$ ), but these were not associated with any serious clinical outcomes. Serious AEs included pulmonary embolism (4\%), back pain (2\%), and diarrhea (2\%). The main reason for study discontinuation in both groups was disease progression (50\% with the combination vs $70 \%$ for letrozole alone). AEs also resulted in dosage interruptions more frequently in the combination $\operatorname{arm}(33 \%$ vs $4 \%)$. There were no treatment-related deaths in either arm and combinatorial therapy was not associated with an increase in pain severity or pain on daily activity.

According to the prescribing information, the recommended starting dose of palbociclib in this setting is 125 $\mathrm{mg}$ once daily with food over a 28-day cycle (21 days on, 7 


\section{Community Translations}

days off). Dose interruptions or delays and/or dose reductions or permanent discontinuation may be required for the management of some AEs, including grade 3 or 4 neutropenia and for coadministration with strong CYP3A inhibitors. The use of strong and moderate CYP3A inducers and strong CYP3A inhibitors should generally be avoided. Palbociclib can cause fetal harm and there are also warnings and precautions about the risk for infection and pulmonary embolism.

The approval of palbociclib and letrozole represents an important advancement for the treatment of ER-positive advanced breast cancer. Palbociclib, marketed as Ibrance by Pfizer, is the first in a new class of targeted therapy to gain regulatory approval and in this indication represents the first front-line treatment option to show improvement over endocrine therapy alone, the standard of care for this patient population. The phase 3 PALOMA-2 trial is currently ongoing (NCT01740427) to determine the effect of this combination on OS, with final data expected sometime in 2016, and several other phase 3 trials of palbociclib in combination with other forms of endocrine therapy are also ongoing.

\section{References}

1. Finn RS, Crown JP, Lang I, et al. The cyclin-dependent kinase $4 / 6$ inhibitor palbociclib in combination with letrozole versus letrozole alone as first-line treatment of oestrogen receptor-positive, HER2 negative, advanced breast cancer. Lancet Oncol. 2015;16:25-35.

2. Ibrance (palbociclib) capsules, for oral use. Prescribing information, Pfizer Inc, February 2015. http://labeling.pfizer.com/ShowLabeling. aspx?id=2191.

3. Koehler M, VanArsdale TL, Shields D, et al. Mechanism of action for combined CDK4/6 and ER inhibition in ER-positive breast cancer. Ann Oncol. 2014;25(Suppl. 1):i21-i22. 